\title{
Toxicity of internalized laser generated pure silver nanoparticles to the isolated rat hippocampus cells
}

Toxicology and Industrial Health 2017, Vol. 33(7) 555-563

(C) The Author(s) 2017

Reprints and permissions:

sagepub.co.uk/journalsPermissions.nav DOI: $10.1177 / 0748233717690992$ journals.sagepub.com/home/tih (S)AGE

\author{
Canan Kursungoz ${ }^{1,2}$, Sadık Taşkın Taş ${ }^{3}$, \\ Mustafa F Sargon ${ }^{4}$, Yıldırım Sara ${ }^{3}$, and Bülend Ortaç ${ }^{1,2}$
}

\begin{abstract}
Silver nanoparticles (AgNPs) are the most commonly used nanoparticles (NPs) in medicine, industry and cosmetics. They are generally considered as biocompatible. However, contradictory reports on their biosafety render them difficult to accept as 'safe'. In this study, we evaluated the neurotoxicity of direct AgNP treatment in rat hippocampal slices. We produced pure uncoated AgNPs by a pulsed laser ablation method. NP characterization was performed by Ultraviolet (UV) visible spectrophotometer, scanning electron microscope, transmission electron microscope (TEM) and energy-dispersive X-ray spectroscopy. Rat hippocampal slices were treated with AgNPs for an hour. AgNP exposure of hippocampal tissue resulted in a significant decrease in cell survival in a dose-dependent manner. Our TEM results showed that AgNPs were distributed in the extracellular matrix and were taken into the cytoplasm of the neurons. Moreover, we found that only larger AgNPs were taken into the neurons via phagocytosis. This study showed that the pure AgNPs produced by laser ablation are toxic to the neural tissue. We also found that neurons internalized only the large NPs by phagocytosis which seems to be the major mechanism in AgNP neurotoxicity.
\end{abstract}

\section{Keywords}

Laser ablation, silver nanoparticles, classical endocytosis, internalization of nanoparticles, hippocampus, neurodegenerative disease

Received 19 August 2016; Revised 27 December 2016; Accepted 28 December 2016

\section{Introduction}

Nanometre scale provides materials with unique physicochemical properties that are completely different than their bulk counterparts. Although some materials show minimum risks for the human health due to their biochemically inert and biocompatible nature, they might exhibit certain levels of toxicity in nanometre scales due to their high surface-to-volume ratio and high surface reactivity, especially when they encounter biological molecules.

Nanoparticles (NPs) are used in a number of different applications in medical, biological, electronic and industrial fields. One of the most commonly used types of NPs in those applications is the silver nanoparticles (AgNPs). They are used as therapeutics; cosmetics; and antimicrobial coatings on medical implants, catheters, wound dressings and so on (Chen and Schluesener, 2008). AgNPs are also utilized as imaging agents because of their unique plasmon-resonance optical scattering properties (Farokhzad et al., 2006). Central nervous system has a high risk of NP exposure since NPs can reach the brain both by systemic circulation and through the

\footnotetext{
' UNAM - National Nanotechnology Research Center, Bilkent University, Ankara, Turkey

${ }^{2}$ Institute of Materials Science and Nanotechnology, Bilkent University, Ankara, Turkey

${ }^{3}$ Department of Medical Pharmacology, Hacettepe University, Ankara, Turkey

${ }^{4}$ Department of Anatomy, Faculty of Medicine, Hacettepe University, Ankara, Turkey
}

\section{Corresponding author:}

Bülend Ortaç, UNAM - National Nanotechnology Research Center, Bilkent University, 06800 Ankara, Turkey; Institute of Materials Science and Nanotechnology, Bilkent University, 06800 Ankara, Turkey.

Email: ortac@unam.bilkent.edu.tr 
olfactory bulb via the olfactory mucosa (Bruchez $\mathrm{Jr}$ et al., 1998, Oberdörster et al., 2004). It was shown that AgNPs are detectable in the brain after inhalation (Hoet et al., 2004). Hippocampus was chosen for our study since it is the major brain region responsible for learning and memory. Moreover, hippocampus was shown to be highly susceptible to cell death (Riva et al., 2012).

The cellular effects of AgNPs were studied in various mammalian cell lines in in vitro settings (BraydichStolle et al., 2005; Burd et al., 2007; Hussain et al., 2005). AgNPs were shown to be toxic to mitochondria (Hussain et al., 2006) due to oxidative stress leading to inflammation, deoxyribonucleic acid (DNA) damage and finally apoptosis (Ahamed et al., 2010). It is known that AgNPs can accumulate in the brain (Sung et al., 2008) and damage learning and memory retention via reactive oxygen species (ROS) in rats (Liu et al., 2012). Furthermore, AgNPs alter the expression of genes related to motor neuron disorders, neurodegenerative diseases and immune function (Li et al., 2003). Although AgNPs have been considered as safe and commonly used in drugs, cosmetics and so on for decades, recent studies suggest that they would lead to serious toxicity in the nervous system as well as other systems.

In order to exert intracellular toxic effects, NPs should be taken into the cell and interact with macromolecules. Cellular uptake mechanisms for NPs are various forms of endocytosis depending on their physicochemical properties (Saptarshi et al., 2013). Surface chemistry is the main determinant of NP toxicity (Lu et al., 2010). So far, studies on AgNPs have all used coated NPs (polyvinylpyrrolidone, antibodies, surfactant or chemical byproducts) resulting in different surface chemistries while uncoated pure AgNP toxicity is yet to be elucidated. Since pure uncoated AgNPs are the most common AgNPs in nature and in industry as products or byproducts such as during laser ablation, it is important to determine their mechanism for internalization which is correlated with their toxicity (Barcikowski et al., 2009).

In this study, we produced pure uncoated AgNPs without any chemical surface modifications using a laser ablation method and aimed to investigate the direct toxicity of the AgNPs to the neural tissue and their mechanism of internalization by the neurons in rat hippocampal slices.

\section{Methods}

\section{Animals and hippocampal slice preparation}

Three-week old male Wistar albino rats were used for the procedures involving hippocampal slices. After anaesthetizing by diethyl ether, the animals were rapidly decapitated, hippocampi were removed and transverse slices at a thickness of $400 \mu \mathrm{m}$ were cut in oxygenated, $50 \mathrm{ml}$ of ice-cold dissection buffer (low $\mathrm{Ca}^{2+} /$ high $\mathrm{Mg}^{2+}$ artificial cerebrospinal fluid (ACSF)), using a vibroslice (World Precision Instruments, Sarasota, Florida, USA). Oxygen $\left(\mathrm{O}_{2}\right)$ was supplied using a $95 \% \mathrm{O}_{2}$ and $5 \%$ carbon dioxide $\left(\mathrm{CO}_{2}\right)$ gas mixture (carbogen).

All animal experiments were approved by Hacettepe University Animal Experimentations Local Ethics Board (number: B.30.2.HAC.0.05.06.00/50).

\section{NP production and characterization}

A $99.99 \%$ pure silver ( $\mathrm{Ag})$ block was used as the target of a laser beam to produce the AgNPs using a laser ablation method as described previously (Alkis et al., 2012). NPs were produced by a commercial nanosecond pulsed Neodymium-doped yttrium lithium fluoride (Nd:YLF) laser (Empower Qswitched laser; Spectra Physics, Santa Clara, CA, USA) operating at $527 \mathrm{~nm}$ with 100 -ns pulse duration and $16-\mathrm{W}$ average output power at $1-\mathrm{kHz}$ pulse repetition rate corresponding to a pulse energy of $16 \mathrm{~mJ}$. The Ag block was placed in a glass vessel containing $20 \mathrm{ml}$ of pure deionized water. The laser beam was focused on the target by the help of a plano-convex lens with a focal length of $50 \mathrm{~mm}$. The height of liquid layer over the target was approximately $5 \mathrm{~mm}$. The laser ablation was carried out for $5 \mathrm{~min}$ by the beam scanning over the target surface, creating a solution with dispersed NPs in the liquid medium. The Ag block was weighed before and after the ablation process, and the concentration of the AgNP solution was determined to be $0.1 \mathrm{mg} / \mathrm{ml}$.

UV-Visible-Near Infrared (UV-Vis-NIR) spectrophotometer (Varian/Cary 5000; Agilent Technologies, Santa Clara, CA, USA) analysis was performed for the determination of characteristic absorbance spectrum of the AgNPs. The structural and topographical properties of AgNPs were analysed using a scanning electron microscope (SEM; FEI Quanta 200 FEG, Hillsboro, OR, USA). Further morphological analysis of the AgNPs was done using a transmission electron microscope (TEM; FEI Tecnai G2F30, Hillsboro, OR, USA) together with the elemental characterizations using energy-dispersive X-ray (EDX) analysis. TEM samples were prepared by dropping the AgNP solution onto a carbon-coated TEM grid. 


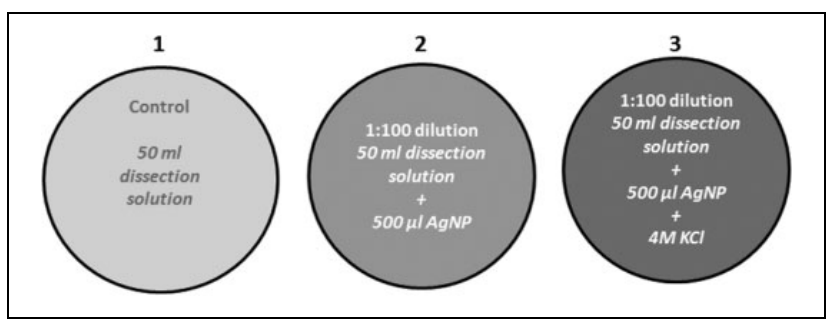

Figure I. Rat hippocampal slice incubation with pure AgNPs. Control slices were incubated with dissection buffer solution as shown in condition I. For the experimental group, condition 2 included 500- $\mu \mathrm{l} \mathrm{AgNP}$ and $50-\mathrm{ml}$ dissection buffer solution to achieve $1: 100$ dilution and condition 3 included additional $4-\mathrm{M} \mathrm{KCl}$ for high $\mathrm{K}^{+}$administration to check whether the AgNPs are located in the presynaptic vesicles of hippocampal neurons. AgNP: silver nanoparticle; $\mathrm{KCl}$ : potassium chloride.

\section{Administration of AgNPs to hippocampal slices for TEM analysis}

After hippocampal slice preparation, obtained slices were incubated at $37^{\circ} \mathrm{C}$ in ACSF for $60 \mathrm{~min}$ with three different incubation environments as shown in Figure 1. The ACSF solution containing (in $\mathrm{mM}$ ): 124 sodium chloride, 5 potassium chloride $(\mathrm{KCl}), 12$ monosodium phosphate, 26 sodium bicarbonate, $10 \mathrm{D}$-glucose, 2 calcium chloride and 1 magnesium chloride was oxygenated using $95 \% \mathrm{O}_{2}$ and $5 \%$ $\mathrm{CO}_{2} . \mathrm{KCl}$ with a final concentration of $4 \mathrm{M}$ was added into one of the AgNP incubation solutions to check whether the AgNPs are located in the presynaptic vesicles in hippocampus cells. It is known that high $\mathrm{K}^{+}$concentration leads to stimulated synaptic vesicular recycling; thus it helps to demonstrate the AgNPs in the presynaptic vesicles, if any (Sara et al., 2002).

\section{Sample preparation and TEM analysis of the hippocampal slices}

The incubated hippocampal slices were fixed in $2.5 \%$ gluteraldehyde for $24 \mathrm{~h}$. Then, the samples were washed in Sorenson's phosphate buffer (SPB, pH: 7.4) and postfixed in $1 \%$ osmium tetroxide. Following this step, the samples were rewashed using SPB to reach the dehydration step. The samples were dehydrated using increasing concentrations of alcohol (25\%, 50\%, $75 \%$ and pure alcohol). Then, the tissues were washed with propylene oxide twice and embedded in the media which were prepared by mixing 1:1 propylene oxide and epoxy resin embedding material. The samples were incubated in this mixture for $1 \mathrm{~h}$, and at the end of the incubation period, the same amount of epoxy resin embedding material was added to the mixture and the ratio of the mixture was increased to $1 / 3$. The samples were incubated in the rotator overnight in the resulting mixture and the preparation for the embedding part was completed. The samples were incubated in $60^{\circ} \mathrm{C}$ incubator for $48 \mathrm{~h}$ after being embedded into plastic capsules with the epoxy resin embedding material. At the end of $48 \mathrm{~h}$, the samples were taken out of the incubator, and semithin sections about $2 \mu \mathrm{m}$ in thickness were cut by ultramicrotome (LKB-Nova, Sweden). The sections were stained with methylene blue and examined by an Optiphot (Nikon, Japan) light microscope to determine the area to be sectioned further. After trimming procedure of these semithin sections, ultrathin sections at a thickness of $60 \mathrm{~nm}$ were taken by ultramicrotome, collected on copper $(\mathrm{Cu})$ grids, stained with uranyl acetate and lead citrate and examined by a TEM (JEOL JEM 1200 EX, Japan).

\section{Cell viability assay}

For the investigation of cell viability, we have chosen five different AgNP concentrations: 10, 15, 20, 25 and $30 \mathrm{mg} / \mathrm{L}$. Control slices were incubated without NPs in ACSF solution. All groups were oxygenated with carbogen mixture except a second NP-free group which was treated with hypoxic conditions as the positive control group to assess the baseline metabolic activity. After incubating the hippocampal slices with the AgNPs for $60 \mathrm{~min}$, the slices were removed from the incubation solution and further incubated with $0.5-\mathrm{mg} / \mathrm{ml} 3-(4,5-$ dimethylthiazol-2-yl)-2,5-diphenyltetrazolium bromide (MTT, Sigma Aldrich, Germany) solution for $60 \mathrm{~min}$ at $37^{\circ} \mathrm{C}$. Then, $4-\mathrm{mM}$ acidified isopropanol was added to the solution to solubilize the formazan crystals formed at the end of the reaction. The absorbance was measured at $570 \mathrm{~nm}$ using a microplate reader (Synergy HT; BioTek, Winooski, Vermont, USA).

\section{Statistical analysis}

The statistical analysis of MTT cell viability assay was performed by one-way analysis of variance with post hoc Tukey test to compare the control group to the experimental groups. A $p$ value below 0.05 was accepted statistically significant. 


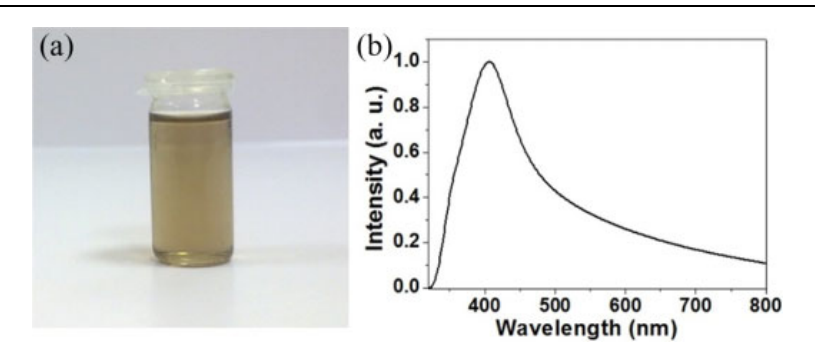

Figure 2. Optical properties of pure AgNPs. (a) The image of $0.1-\mathrm{mg} / \mathrm{ml} \mathrm{AgNP}$ solution produced by a laser ablation method. No precipitation was detected for at least for 4 months. (b) Absorption spectrum exhibited absorbance peak at $406 \mathrm{~nm}$ corresponding to AgNP. AgNP: silver nanoparticle.

\section{Results}

\section{NP production and characterization}

The AgNPs were produced in deionized water at a concentration of $0.1 \mathrm{mg} / \mathrm{ml}$, and the production was observed as the change of colour to yellow appearance (Figure 2(a)). The optical absorption spectrum of AgNPs was determined by UV-Vis absorption spectroscopy. Figure 2(b) shows that the optical absorption spectrum of AgNPs giving the specific Ag peak at $406 \mathrm{~nm}$, which is consistent with the literature (Rai et al., 2009).

First, we evaluated the size distribution of NPs produced by laser ablation method from pure Ag. In SEM images (Figure 3(a)), most of the AgNPs were found to be smaller than $100 \mathrm{~nm}$ in diameter but there were also larger AgNPs that were up to approximately $500 \mathrm{~nm}$ in diameter. The NPs were also shaped spherically. The TEM analysis was performed to further characterize the AgNPs and determine their structure (Figure 3(b)). The TEM results confirmed the SEM results in terms of shape and size of the AgNPs. Furthermore, the NPs showed no aggregation pattern in the TEM images. The TEM images also demonstrated that the AgNPs had a crystal structure.

EDX analysis was performed for further confirmation of the purity of AgNP solution. As shown in the inset of Figure 3(b), the solution was consisting of carbon, $\mathrm{Cu}$ belonging to the TEM grids and $\mathrm{Ag}$ as can be determined from the peaks corresponding to those elements. Thus, there was no interference of any impurity during the AgNP administration to the hippocampus tissue.

\section{TEM analysis of the hippocampus tissue samples}

Electron microscopy was performed to visualize the distribution of AgNPs in extracellular and intracellular areas of the neural tissue. TEM analysis displayed that the AgNPs were distributed both in the extracellular and the intracellular matrices (Figure 4(b) to (d)). Additionally, we never observed the presence of AgNPs inside a specific organelle; rather, they were always distributed in the cytoplasm. Small AgNPs below $100 \mathrm{~nm}$ in diameter were only found in the extracellular matrix while larger NPs were present both in extracellular and intracellular regions. Moreover, phagocytosis of an AgNP by a hippocampal neuron was also observed and photographed in the TEM analysis (Figure 4(e)).

\section{Cell viability assay}

In order to evaluate the toxicity of pure uncoated AgNPs of different sizes, we employed MTT cell viability assay. The MTT analysis showed a dosedependent toxicity for AgNPs. AgNP administration at 10,15 and $20 \mathrm{mg} / \mathrm{L}$ did not cause any significant decrease in cell survival while AgNP at 25 and $30 \mathrm{mg} / \mathrm{L}$ led to a statistically significant cell death $(p<0.0001)$ when compared to the control group (Figure 5).

\section{Discussion}

To the best of our knowledge, this is the first study evaluating the toxicity of the pure uncoated AgNPs produced by a laser ablation method by administration to the hippocampal tissue. In this study, (i) AgNP treatment caused cell death in a dose-dependent manner, (ii) pure AgNPs were localized in the cytoplasm rather than organelles, (iii) AgNPs were taken up by hippocampal neurons via phagocytosis, (iv) this endocytosis pathway was separate from the vesicular recycling pathway since stimulation of vesicular recycling did not increase the AgNP uptake and (v) this endocytosis pathway preferentially selected the large AgNPs.

\section{Produced uncoated pure AgNPs were spherical and did not aggregate}

The broad size distribution of NPs is considered as a drawback of the laser ablation method in the NP synthesis. To control the size, surfactant materials are used, so that the NPs can be produced in a specified size range (Mafune et al., 2000). However, this would affect the purity and most probably change the behaviour of the AgNPs in the biological environment. For this study, purity was a significant parameter to 

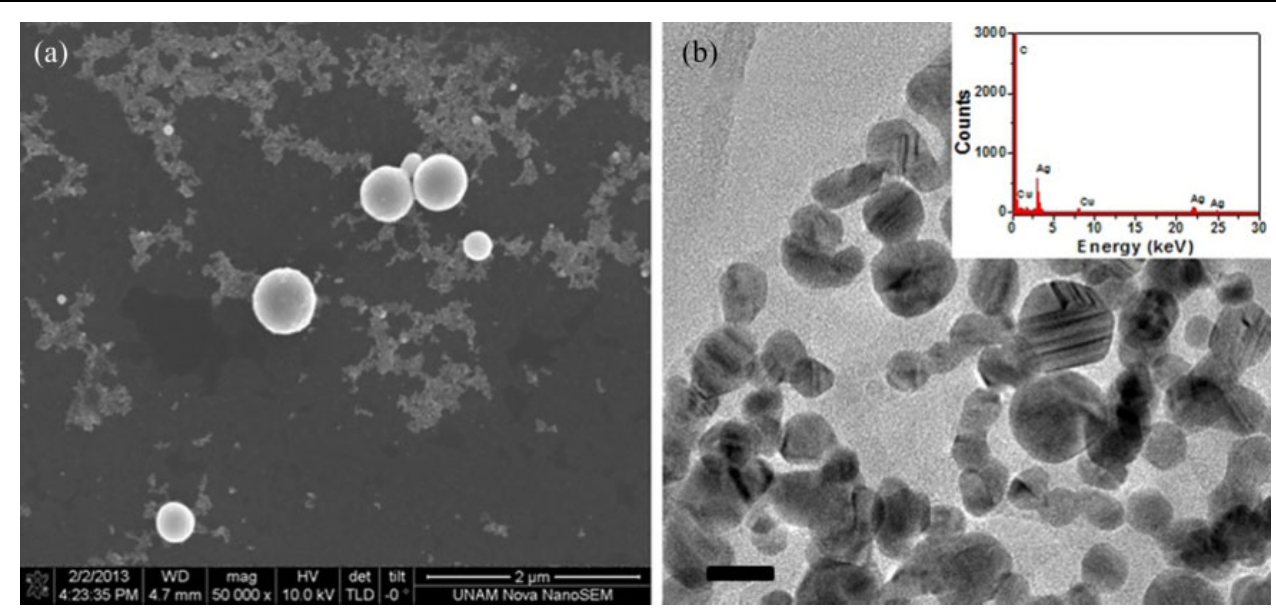

Figure 3. Representative SEM and TEM images. (a) SEM image of AgNPs (2- $\mu \mathrm{m}$ scale bar). (b) TEM image of the AgNPs (20-nm scale bar); the inset corresponds to EDX analysis of the AgNP sample. The solution consisted of $\mathrm{C}$ and $\mathrm{Cu}$, which belonged to TEM grids, and $\mathrm{Ag}$ as can be determined from the peaks corresponding to those elements, indicating no interference of any other impurity in AgNP solution. SEM: scanning electron microscope; TEM: transmission electron microscope; AgNP: silver nanoparticle; EDX: energy-dispersive X-ray; C: carbon; Cu: copper; Ag: silver.

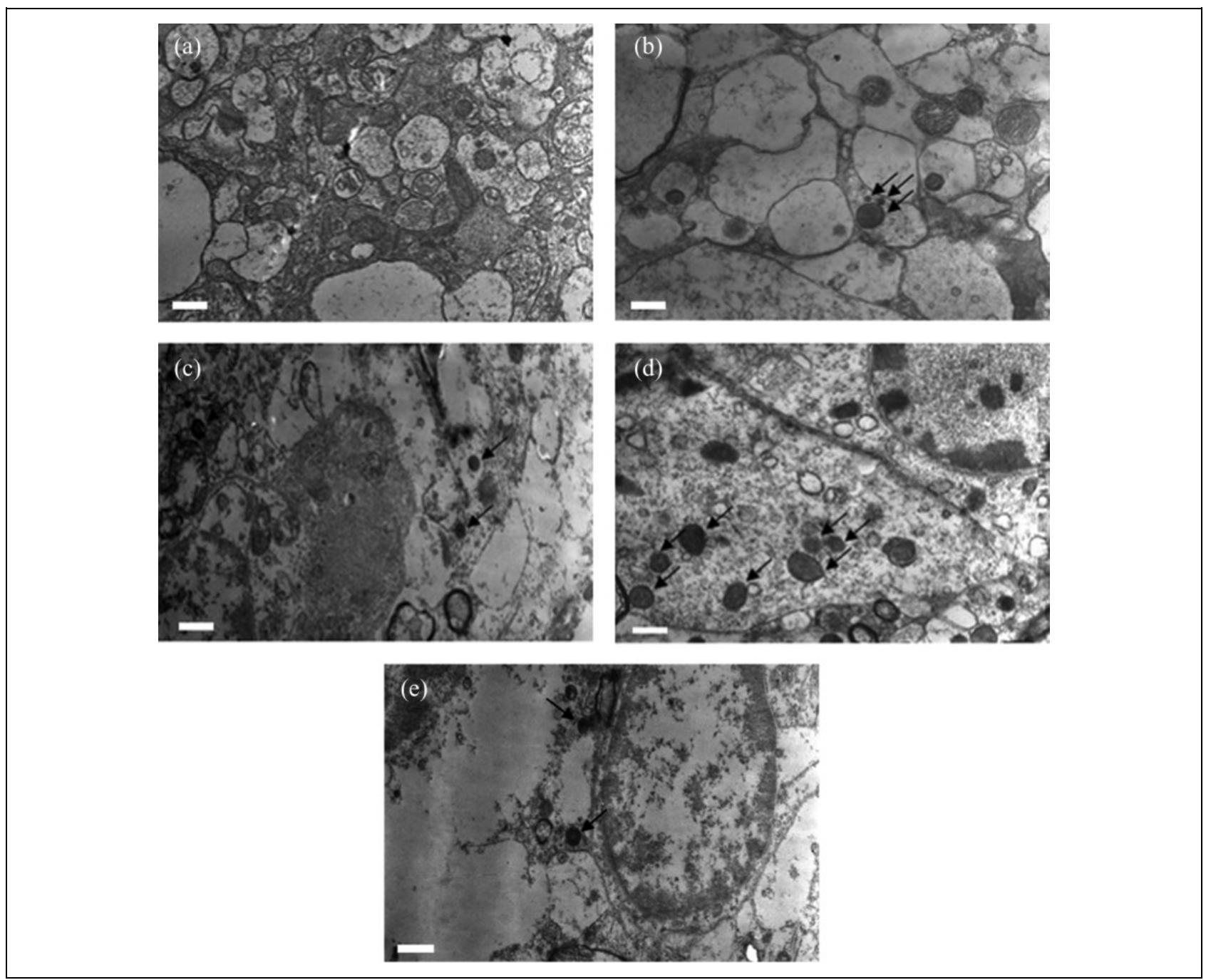

Figure 4. TEM images showing AgNP distribution in hippocampal slice treated with AgNPs, (a) control; (b) and (c) AgNPs in the extracellular region; (d) AgNPs inside the cell, distributed in the cytosol; (e) phagocytosis of AgNPs. Arrows indicate the AgNPs. Scale bar for each figure is $500 \mathrm{~nm}$. TEM: transmission electron microscope; AgNP: silver nanoparticle. 


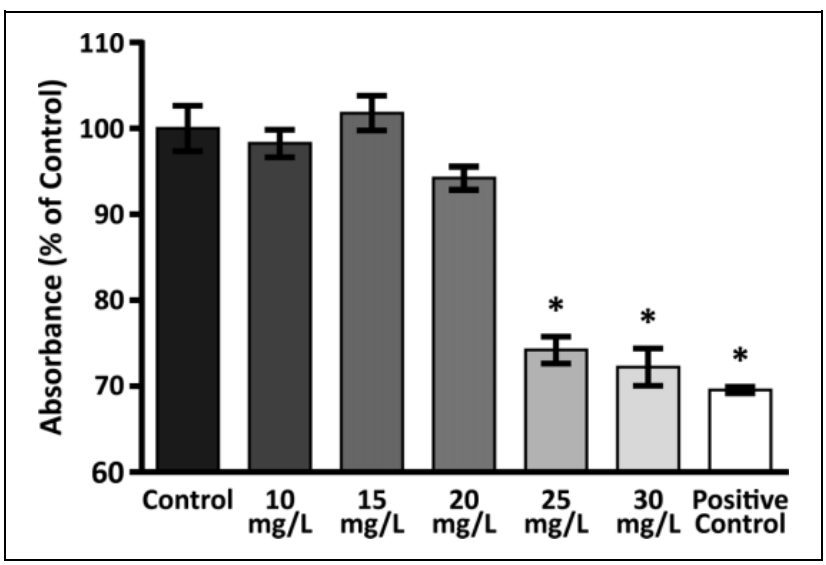

Figure 5. MTT cell viability assay results of hippocampal cells upon exposure to various AgNP concentrations for I h; 10-, I5- and 20-mg/L AgNP treatment did not cause a significant decrease in cell survival. One hour of 25- and 30-mg/L AgNP incubation resulted in cellular toxicity and cell death, which was found to be statistically significant $(p<0.000 \mathrm{I})$. Control group was incubated in physiological conditions while the positive control group did not receive oxygenation during incubation. MTT: 3-(4,5-dimethylthiazol-2-yl)-2,5-diphenyltetrazolium bromide; AgNP: silver nanoparticle.

determine the AgNP localization in the hippocampal tissue, therefore we avoided using surfactants. Furthermore, the broad size distribution obtained by the laser ablation method is an advantage to mimic the industrially occurring NPs in real-life settings.

It was shown that the shape of the NPs is strongly correlated with their cellular uptake rates, with rodshaped NPs show the highest uptake rate, followed by spheres, cylinders and finally cubes (Gratton et al., 2008). We assume that production of spherical NPs by the laser ablation method increased their phagocytosis.

Besides the importance of shape and size in cellular uptake, aggregation is an important feature that NPs exhibit and generally a number of nanostructures exert their toxic effects upon aggregation ( $\mathrm{Nel}$ et al., 2006). In this study, aggregation was not observed in TEM results before tissue administration, thus, whether the AgNPs would aggregate or not depended on their behaviour inside the tissue.

\section{Rat hippocampal neurons internalize AgNPs by phagocytosis}

Our TEM results of the tissues exhibited the distribution of large size NPs both in intracellular and extracellular regions. Small AgNPs below $100 \mathrm{~nm}$ in size were found to be exclusively localized in the extracellular region, showing that the internalization mechanism solely works for NPs larger than a specific size. Furthermore, the internalized NPs were observed only in the cytoplasms of the hippocampal cells and not in the organelles. Previous studies on the cellular localization of AgNPs in tissues other than hippocampus show that these NPs can be found in membrane-bound organelles. One group showed that the starch-coated AgNPs were taken into glioblastoma cells and were localized in the endosomes and lysosomes (AshaRani et al., 2009). In our study, these AgNPs were not found in endosomes. This might be due to the endosomal escape phenomenon, in which NPs tend to escape the endosomes or lysosomes and are distributed in the cytoplasm freely (Panyam et al., 2002). Alternatively, our pure NPs may not be subject to the same uptake and distribution mechanisms as coated NPs since NPs bind to different sets of proteins and lipids based on their coatings.

\section{Neurons preferentially phagocytose large AgNPs}

Preferential uptake of large AgNPs into the cell cytoplasm might be explained with the internalization pathway. So far, five endocytosis pathways were discovered. They are kiss-and-run mechanism, clathrinmediated endocytosis, phagocytosis, caveolae and macropinocytosis. Kiss-and-run mechanism and clathrin-mediated endocytosis pathways take part in sustaining the vesicular neurotransmitter release and vesicle recycling in the synapse. Phagocytosis does not have a role in neurotransmitter turnover but it can internalize particles larger than $100 \mathrm{~nm}$, which cannot be internalized by other mechanisms. We did not observe the presence of NPs in axon terminals in conditions where the synaptic vesicular turnover either was kept at the basal rate or was significantly increased by high $\mathrm{K}^{+}$treatment, which is known to stimulate the vesicular recycling in the synapses (Sara et al., 2002). Rather, AgNPs were located inside the neuron soma. Our data revealed that the AgNP internalization process was via outward membrane projections in the soma region, implying that vesicular recycling pathways do not have a role in NP internalization, at least for this size set of pure uncoated AgNPs.

We excluded clathrin-mediated endocytosis pathway as a major mechanism of AgNP cellular entry for the reasons listed below. First, the size of the AgNP 
was greater than the average size of molecules can be handled by clathrin pathway, which is around $200 \mathrm{~nm}$ (Rejman et al., 2004). Second, we did not observe a characteristic dense area around the internalized $\mathrm{AgNP}$ as an indicator of clathrin coating. Lastly, we observed an extracellular elongation of the membrane as if it was engulfing the AgNP.

The findings of this study suggest phagocytosis as the internalization mechanism for pure uncoated AgNPs rather than any other endocytosis pathways. This uptake mechanism is crucial for further toxicology studies since it may be a novel entry mechanism for NPs that do not have altered surface chemistry or coating. There are studies about the uptake mechanisms of AgNPs into the cells, but to our knowledge, this is the first time that pure uncoated AgNPs produced by laser ablation are shown to be taken into neurons in the isolated hippocampus tissue. Our study proves that even a pure NP would facilitate the cellular uptake and it can be internalized and localized in the cell. These findings indicate the phagocytosis pathway might be the cellular uptake mechanism for the pure AgNPs, rather than other endocytosis pathways.

\section{AgNP treatment dose-dependently causes cell death}

The dose selection for MTT cell viability assay was performed according to the literature. A wide range of AgNP doses were administered to cells in vitro in a number of studies (Hussain et al., 2005; Kawata et al., 2009; Kim et al., 2012). Accordingly, we wanted to determine the effects of the lowest possible dose which would not affect the hippocampal cells and the highest dose which would lead to death of cells as much as hypoxic conditions. The doses administered by Hussain et al. (2005) to the rat liver cells were used as a reference, and $10 \mathrm{mg} / \mathrm{L}$ was found to not affect the hippocampal cell survival. Moreover, $30 \mathrm{mg} / \mathrm{L}$ caused cell death comparable to the hypoxic positive group. Thus, to observe the dose dependence of cell death upon AgNP administration, we performed cell viability analysis with five doses between $10 \mathrm{mg} / \mathrm{L}$ and $30 \mathrm{mg} / \mathrm{L}$.

Heavy metal toxicity is generally associated with cognitive and motor function disorders. Since hippocampus is a major brain region responsible for learning and memory and it is highly susceptible to cell death (Riva et al., 2012), we selected this region for our experiments.
AgNP incubation decreased cell survival in a dosedependent manner in rat hippocampal slices. Low doses of AgNP did not trigger any considerable decrease in cell survival while higher doses of AgNP resulted in a significant cell death compared to the controls. However, we did not perform a timedependent assay to determine the toxicity of the lower doses in long-term periods, which might be considered as a limitation of the study. Therefore, lower doses of AgNPs might also be toxic for the neural tissue in long-term exposure. Our data are also consistent with a recent study which demonstrated that AgNP results in cellular toxicity in HT22 hippocampus cell line in a dose-dependent manner (Ma et al., 2015).

The possible mechanism for AgNP toxicity seems to be related to an increase in the ROS levels that causes oxidative stress on the cells. Oxidative stress is seen when the anti-oxidant defence mechanism cannot compete with the increasing ROS levels (Nel et al., 2006). A number of studies showed that the AgNPs cause lipid peroxidation in cell membrane, increased ROS levels and oxidative stress which result in DNA damage and apoptosis (Gopinath et al., 2010). AgNPs were also shown to upregulate the oxidative stress-related genes in the caudate nucleus, frontal cortex and hippocampus regions of brain in mice (Rahman et al., 2009). Thus, it is crucial to determine the exact localization of AgNPs in the cells following exposure. While we did not evaluate the mechanism of AgNP-induced cellular toxicity, we speculate that the toxicity demonstrated with cell viability assay might be the result of ROS production in the hippocampal cells, leading to cell death on the basis of previous research.

\section{Conclusion}

In this study, we showed the distribution and the toxicity of the pure AgNPs produced by the laser ablation method in the rat hippocampal slices. Moreover, we demonstrated that the cellular uptake mechanism of pure AgNPs depends on their sizes. Since we did not observe the presence of AgNPs in synaptic vesicles following stimulation of synaptic vesicular recycling, we concluded that phagocytosis is the type of endocytotic pathway that dominates the entry of pure AgNPs into the hippocampal neurons. Our data provide significant information for the further cellular and molecular research about the neurotoxicology of NPs since exposure to AgNPs might result in 
neurodegeneration. Furthermore, this study showed that laser ablation is a considerably useful method for studying NP toxicity, since it provides pure NPs mimicking the ones encountered in the industry.

\section{Acknowledgements}

The authors would like to thank TÜBİTAK and TÜBAGEBIP for their financial support.

\section{Declaration of Conflicting Interests}

The author(s) declared no potential conflicts of interest with respect to the research, authorship, and/or publication of this article.

\section{Funding}

The author(s) disclosed receipt of the following financial support for the research, authorship, and/or publication of this article: This project was supported by TÜBITAK grant 113 S223 and partially supported by TÜBA-GEBIP.

\section{References}

Ahamed M, Siddiqui MA, Akhtar MJ, et al. (2010) Genotoxic potential of copper oxide nanoparticles in human lung epithelial cells. Biochemical and Biophysical Research Communications (Elsevier Inc) 396(2): 578-583.

Alkis S, Alevli M, Burzhuev S, et al. (2012) Generation of InN nanocrystals in organic solution through laser ablation of high pressure chemical vapor deposition-grown InN thin film. Journal of Nanoparticle Research 14(8): 1048.

AshaRani PV, Mun GLK, Hande MP, et al. (2009) Cytotoxicity and genotoxicity of silver nanoparticles in human cells. ACS Nano 3(2): 279-290.

Barcikowski S, Hahn A, Walter J, et al. (2009) Health risks of nanoparticulate emissions during femtosecond and picosecond pulsed laser machining. SPIE LASE: Lasers and Applications in Science and Engineering. International Society for Optics and Photonics 7201: 1-6.

Braydich-Stolle L, Hussain S, Schlager JJ, et al. (2005) In vitro cytotoxicity of nanoparticles in mammalian germline stem cells. Toxicological Sciences 88(2): 412-419.

Bruchez M Jr., Moronne M, Gin P, et al. (1998) Semiconductor nanocrystals as fluorescent biological labels. Science 281(5385): 2013-2016.

Burd A, Kwok CH, Hung SC, et al. (2007) A comparative study of the cytotoxicity of silver-based dressings in monolayer cell, tissue explant, and animal models. Wound Repair and Regeneration 15(1): 94-104.

Chen X and Schluesener HJ (2008) Nanosilver: a nanoproduct in medical application. Toxicology Letters 176(1): $1-12$.
Farokhzad OC, Cheng J, Teply BA, et al. (2006) Targeted nanoparticle-aptamer bioconjugates for cancer chemotherapy in vivo. Proceedings of the National Academy of Sciences of the United States of America 103(16): 6315-20.

Gopinath P, Gogoi SK, Sanpui P, et al. (2010) Signaling gene cascade in silver nanoparticle induced apoptosis. Colloids and Surfaces B: Biointerfaces, Elsevier BV 77(2): 240-245.

Gratton SEA, Ropp PA, Pohlhaus PD, et al. (2008) The effect of particle design on cellular internalization pathways. Proceedings of the National Academy of Sciences of the United States of America 105(33): 11613-11618.

Hoet PH, Brüske-Hohlfeld I and Salata OV (2004) Nanoparticles - known and unknown health risks. Journal of Nanobiotechnology 2: 12.

Hussain SM, Hess KL, Gearhart JM, et al. (2005) In vitro toxicity of nanoparticles in BRL 3A rat liver cells. Toxicology in Vitro 19(7): 975-983.

Hussain SM, Javorina AK, Schrand AM, et al. (2006) The interaction of manganese nanoparticles with PC-12 cells induces dopamine depletion. Toxicological Sciences 92(2): 456-463.

Kawata K, Osawa M and Okabe S (2009) In vitro toxicity of silver nanoparticles at noncytotoxic doses to HepG2 human hepatoma cells. Environmental Science and Technology 43(15): 6046-6051.

Kim TH, Kim M, Park HS, et al. (2012) Size-dependent cellular toxicity of silver nanoparticles. Journal of Biomedical Materials Research - Part A 100(4): 1033-1043.

Li N, Sioutas C, Cho A, et al. (2003) Ultrafine particulate pollutants induce oxidative stress and mitochondrial damage. Environmental Health Perspectives 111(4): 455-460.

Liu Y, Guan W, Ren G, et al. (2012) The possible mechanism of silver nanoparticle impact on hippocampal synaptic plasticity and spatial cognition in rats. Toxicology Letters, Elsevier Ireland Ltd 209(3): 227-231.

Lu W, Senapati D, Wang S, et al. (2010) Effect of surface coating on the toxicity of silver nanomaterials on human skin keratinocytes. Chemical Physics Letters, Elsevier BV 487(1-3): 92-96.

Ma W, Jing L, Valladares A, et al. (2015) Silver nanoparticle exposure induced mitochondrial stress, caspase-3 activation and cell death: amelioration by sodium selenite. International Journal of Biological Sciences 11(8): 860-867.

Mafune F, Kohno J, Takeda Y, et al. (2000) Formation and size control of sliver nanoparticles by laser ablation in aqueous solution. Journal of Physical Chemistry B 104(39): 9111-9117. 
Nel A, Xia T, Mädler L, et al. (2006) Toxic potential of materials at the nanolevel. Science (New York, N.Y.) 311(February): 622-627.

Oberdörster G, Sharp Z, Atudorei V, et al. (2004) Translocation of inhaled ultrafine particles to the brain. Inhalation Toxicology 16(6-7): 437-445.

Panyam J, Zhou W-Z, Prabha S, et al. (2002) Rapid endolysosomal escape of poly(DL-lactide-co-glycolide) nanoparticles: implications for drug and gene delivery. The FASEB Journal 16(10): 1217-1226.

Rahman MF, Wang J, Patterson TA, et al. (2009) Expression of genes related to oxidative stress in the mouse brain after exposure to silver-25 nanoparticles. Toxicology Letters 187(1): 15-21.

Rai M, Yadav A and Gade A (2009) Silver nanoparticles as a new generation of antimicrobials. Biotechnology Advances, Elsevier Inc 27(1): 76-83.

Rejman J, Oberle V, Zuhorn IS, et al. (2004) Sizedependent internalization of particles via the pathways of clathrin- and caveolae-mediated endocytosis. The Biochemical Journal 377(Pt 1): 159-69.

Riva D, Njiokiktjien C and Bulgheroni S (2012) Brain Lesion Localization and Developmental Functions: Frontal lobes, Limbic system, Visuocognitive System. Paris and London: John Libbey Eurotext.

Saptarshi SR, Duschl A, Lopata AL, et al. (2013) Interaction of nanoparticles with proteins: relation to bio-reactivity of the nanoparticle. Journal of Nanobiotechnology 11(1): 26.

Sara Y, Mozhayeva MG, Liu X, et al. (2002) Fast vesicle recycling supports neurotransmission during sustained stimulation at hippocampal synapses. The Journal of Neuroscience: The Official Journal of the Society for Neuroscience 22(5): 1608-1617.

Sung JH, Ji JH, Yoon JU, et al. (2008) Lung function changes in sprague-dawley rats after prolonged inhalation exposure to silver nanoparticles. Inhalation Toxicology 20(6): 567-574. 